$\chi \omega \dot{\omega} \rho \alpha \cdot R E A M, 15-16,2017 / 2018, p p .487-514$

\title{
CE QUE LES GNOSTIQUES ONT FAIT DU PRINCIPE DU BIEN. LE CAS DE BASILIDE
}

\author{
Izabela Jurasz \\ (Centre Léon Robin, CNRS/Université Paris-Sorbonne)
}

\begin{abstract}
The article examines the metamorphosis of the Platonic Principle of Good in the doctrine of Basilides, the $2^{\text {nd }}$ century Christian gnostic. The Basilidian doctrine represents a radical form of dualism, in which the universe-physical and metaphysical - is born from an encounter between Light (good) and Darkness (evil). In his effort to liberate the Light from all contact with Darkness, Basilide refers to several different mediators (eye, mirror, gleam, desire). Analysing the Basilidian myth in the light of the Platonic writings brings out the paradoxes inherent in any attempts to construct a dualistic metaphysics within the Platonic context.
\end{abstract}

Avec la passion qui depuis des décennies ne semble pas faiblir, les rapports entre le platonisme et le gnosticisme font objet d'un débat académique particulièrement riche. Parmi ses thèmes prioritaires, le plus largement discuté est celui de la genèse de l'Un néoplatonicien. Or, selon la thèse de J.D. Turner, les apports du gnosticisme platonisant auraient joué un rôle prééminent dans la formation du système néoplatonicien et de l'Un en particulier ${ }^{1}$. Dans la mesure où le néoplatonisme se distingue par une lecture à la fois systématique et théologique des dialogues de Platon, les composants de l'Un plotinien seront à identifier dans l'Un du Parménide, dans le Démiurge du Timée et dans l'idée du Bien de la République. Selon les partisans de la thèse de Turner, les relectures des textes platoniciens réalisées dans les milieux gnostiques auraient précédé, sinon influencé, le système élaboré par Plotin. La problématique du présent

1. Les positions de J. Turner sont exposées dans son livre Sethian Gnosticism and platonic tradition, Laval, Leiden, 2001. Pour un résumé et la prise de position par rapport au débat suscité par les thèses de Turner, voir M. Bonazzi, "Platonismo e gnosticismo», in P. Galand - E. Malaspina (dir.), Vérité et apparence. Mélanges en l'honneur de Carlos Lévy, Turnhout, Brepols, 2016, pp. 25-37. 
article fait partie de ce vaste débat, mais dans une perspective différente, parce qu'au centre de notre intérêt se trouve une doctrine gnostique. La question initiale, annoncée dans le titre, concerne la manière dont Basilide et ses disciples ont réinterprété l'idée platonicienne du Bien en vue d'une doctrine caractérisée par le dualisme radical. Au terme de cette analyse, nous espérons apporter quelques observations nouvelles qui concernent le rapport entre la gnose basilidienne et la doctrine de Plotin.

L'idée du rapport entre la doctrine de Basilide et celle de Plotin a déjà été avancée, mais principalement dans les recherches sur les origines de la théologie négative ${ }^{2}$. En dehors de cette problématique, une allusion possible à la doctrine de Basilide a été signalée dans la polémique antignostique du Traité 33, à l'endroit où Plotin parle d'une certaine théorie de l'âme: «Il est également absurde d'introduire, comme ils le font, cette autre âme qu'ils composent à partir des éléments.» ${ }^{3}$ Il est difficile de préciser de quelle doctrine gnostique il est question, mais les basilidiens font partie des destinataires potentiels de cette critique. Peut-on trouver encore d'autres critiques qui leur soient adressées, étant donné qu'ils professent une doctrine qui oppose l'intelligible au sensible et attribue à ce dernier l'origine du mal? - voici la question à laquelle nous essaierons de répondre.

Basilide fut un théologien chrétien, actif à Alexandrie dans les premières décennies du $\mathrm{II}^{\mathrm{e}}$ siècle et probablement aussi le premier à se donner le nom de gnostique. Sa doctrine, élaborée sous forme d'exégèse des textes bibliques, nous est connue à travers des témoignages provenant de la littérature hérésiologique ${ }^{4}$.

2. Sur le rapport entre Basilide et la théologie négative de Plotin, voir J. Whittaker, «Basilides on the Ineffability of God», HThR 62 (1969), pp. 367-371; M. Jufresa Muños, «Basilides, a path to Plotinus», Vigiliae Christianae 35 (1981), pp. 5-15.

3. Traité 33 (II 9) 5, 16-17: 'À

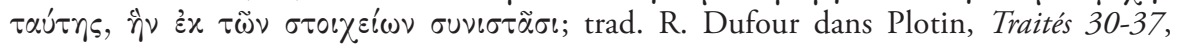
traduction sous la direction de L. Brisson et J.F. Pradeau, Paris, Flammarion, 2006, p. 208. Dans la note explicative, R. Dufour signale plusieurs doctrines gnostiques susceptibles d'être visées par cette remarque. Outre Isidore, fils de Basilide, elle peut s'adresser aux différents branches des valentiniens; voir Plotin, Traités 30-37, op.cit., p. 254. Le caractère artificiel de cet argument qui sans doute relève d'une généralisation au sujet de plusieurs doctrines, est mis en évidence par K. Alt, Philosophie gegen Gnosis. Plotins Polemik in seiner Schrift II 9, Mainz, F. Steiner, 1990, p. 48.

4. La première édition des fragments basilidiens a été réalisée par A. von Harnack, puis complétée par W. Völker et récemment révisée et commentée par W.A. Löhr. L'édition d'Harnack ne contient ni traduction ni commentaire; voir Geschichte der altchristilichen Literatur bis Eusebius, t. 1, Leipzig, 1893, pp. 157-161. W. Völker a complété cette collection par sept fragments provenant d'Irénée de Lyon et d'Hippolyte' de Rome; voir Quellen zur Geschichte der christlichen Gnosis, Tübingen, 1932, pp. 38-57. L'édition de W.A. Löhr comporte quinze témoignages sur Basilide et dix-neuf fragments de ses écrits, provenant de Clément d'Alexandrie, Eusèbe de Césarée, Origène et des Actes d'Archélaüs. Les notices d'Irénée et d'Hippolyte' font objet d'une étude annexe; voir W.A. Löhr, Basilides und seine 
Or, ces citations révèlent des éléments doctrinaux disparates, dont il est difficile de percevoir la cohérence, même en les assignant aux différentes phases de l'évolution doctrinale 5 . Une importante mise au point dans le débat sur l'authenticité des fragments et leur proximité avec la doctrine primitive de Basilide a été faite par W.A. Löhr qui les classe en trois groupes: (1) les témoignages reflétant l'enseignement de Basilide et de son fils Isidore, conservés par Clément d'Alexandrie, Origène, Eusèbe de Césarée et Hégémonius (Actes d'Archélaüs) ${ }^{6}$; (2) les témoignages influencés par le valentinisme, dépendant de la notice d'Irénée de Lyon, reprise par le Pseudo-Tertullien et Épiphane de Salamine; (3) la notice rédigée par 'Hippolyte' de Rome qui correspond à une phase tardive de la doctrine, très éloignée de sa forme originaire ${ }^{7}$. Cette édition, accompagnée d'un ample commentaire, comporte quinze témoignages et dix-neuf fragments (ceux du premier groupe), en réservant un traitement à part aux notices hérésiologiques d'Irénée de Lyon et d'Hippolyte' de Rome. Bien qu'on regrette ce surcroît de méfiance à l'égard des deux derniers textes, il faut reconnaître que Löhr met en relief les éléments qui les attachent aux fragments

Schule. Eine Studie zur Theologie- und Kirchengeschichte des zweiten Jahrhunderts, Tübingen, 1996; W.A. Löhr, «Basilides», dans DGWE, pp. 164-168.

5. Depuis la moitié du XIX ${ }^{\mathrm{e}}$ siècle, des nombreuses études ont été consacrées à la personne et la doctrine de Basilide. La découverte en 1841 des livres IV-X de la Réfutation d'Hippolyte' de Rome, suivie de celles des Actes d'Archélaüs, constitue le moment crucial dans l'histoire des études basilidiennes. Au sujet des recherches sur Basilide, voir: G. Quispel, "L'homme gnostique. La doctrine de Basilide», Eranos Jahrbuch 16 (1948), pp. 89-139; W.A. Löhr, Eine Studie zur Theologie-und Kirchengeschichte des zweiten Jahrhunderts, Tübingen, 1996, pp. 1-4.

6. Clément d'Alexandrie, Stromate I, 145,6 - 146,4; II, 10,1 - 11,2; II, 27, 2; II, 36, 1; II, 112,1 - 114,2; III, 1-3; IV, 81,1 - 83,1; IV, 86,1; IV, 88,5; IV, 153,4; IV, 162,1; IV, 165,3; V, 3,2-3; V, 74,3; VI, 53,2-5; ExcTheod 28. Les fragments cités par Clément concernent quelques points singuliers de la doctrine concernant principalement la morale chrétienne: le péché, la continence et le mariage, le refus du martyre, le baptême des petits enfants, ainsi que la doctrine de l'âme exposée dans l'écrit d'Isidore, fils de Basilide, De l'âme adventice. Voir: W.A. Löhr, Basilides und seine Schule, pp. 42-254. Clément attribue à Basilide une doctrine dualiste, d'apparence assez banale, où Dieu est opposé au diable. En outre, Clément aborde le rapport de Basilide au texte des évangiles et cite son ouvrage intitulé Exégèses, qui pourrait être aussi bien un commentaire qu'une édition du texte évangélique. Un fragment des Exégèses est transmis par Clément et deux autres par Hégémonius. Selon Origène (Hom.Luc I, 2), Basilide aurait écrit son propre évangile peut-être basé sur celui de Luc. En revanche, 'Hippolyte' le présente comme disciple de l'apôtre Matthieu (Ref. VII, 20).

7. L'authenticité des fragments transmis par 'Hippolyte' a été contestée par H. Stähelin, Die gnostischen Quellen Hippolyts, Leipzig 1890. Déjà E. de Faye s'était opposé à cette théorie et voyait dans la notice d'Hippolyte' une "gnose vulgaire», postérieure à

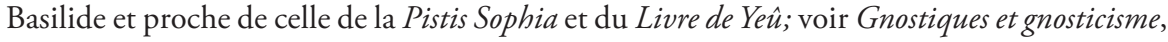
Paris, 1913, p. 215. Encore différent est le jugement de G. Quispel, pour qui la notice d'Hippolyte' représente une "gnose savante»; voir «L'homme gnostique», art. cit., pp. 89-139. 
auxquels il accorde plus de crédit ${ }^{8}$. Notre étude est organisée en fonction d'un texte basilidien, à savoir le long passage provenant des Actes d'Archélaüs, dont nous proposons une relecture à partir de la doctrine platonicienne du Bien, inspirée par les livres VI et VII de la République.

Parmi les passages relatifs à la version primitive de la doctrine prêchée par Basilide, un chapitre de la version latine des Actes d'Archélaüs présente un intérêt majeur pour notre recherche. D'après ce texte, Basilide est un précurseur de Mani et l'auteur d'une doctrine nettement dualiste ${ }^{9}$, construite autour de deux principes contraires, qui sont la lumière et les ténèbres. Évidemment, le contexte polémique des Actes a soulevé des questions au sujet de ce dualisme s'agit-il d'un élément primitif qui a provoqué le rapprochement occasionnel avec Mani ou, au contraire, la doctrine basilidienne, n'étant pas dualiste à l'origine, aurait-elle reçu ce trait pour servir de prolégomènes au manichéisme ${ }^{10}$ ? Hégémonius, l'auteur des Actes d'Archélaüs, situe les débuts de l'activité de Basilide chez les Perses "peu après les temps de nos apôtres» et, dans le dualisme prêché par lui, ne voit qu'une tentative malavisée de paraittre original ${ }^{11}$. Le jugement de la recherche moderne est tout différent. S. Pétrement, dans son travail sur le dualisme platonicien, gnostique et manichéen, présente Basilide comme celui

8. Voir la recension du livre de W.A. Löhr par M.J. Edwards dans Journal of Theological Studies NS 48 (1997), pp. 238-241.

9. Les Actes d'Archélaüs ont été composés avant 350. C'est un dialogue fictif entre l'évêque Archélaüs et Mani. L’idée de présenter un hérétique, Basilide, comme précurseur de Mani, est à rapprocher des Réfutations en prose d'Éphrem le Syrien qui attribue le même rôle à Bardesane, considéré également comme hérétique.

10. Déjà en 1907, W. Bousset a vu dans le fragment des Actes d'Archélaüs une «authentique doctrine» de Basilide et cette position l'a emporté sur les objections - avancées entre autres par G. Quispel; cf. «L'homme gnostique», art. cit., pp. 92-94. Le débat est résumé par W.A. Löhr qui fait de ce passage le fragment 19 du dossier basilidien; voir W.A. Löhr, Basilides und seine Schule, op. cit., pp. 219-248.

11. Le commentaire qui précède le fragment de l'enseignement basilidien est pris en compte par W. Löhr, Basilides und seine Schule, op.cit., pp. 219; 223-225. Nous en donnons ici la traduction: "Il fut chez les Perses un prédicateur nommé Basilide qui aussi vécut auparavant, peu après les temps de nos apôtres. Lui, étant astucieux et ayant constaté qu'à son époque tout avait été déjà formulé, a voulu prêcher le dualisme, ce que faisait aussi Scythianus.» (Actes d'Archélaüs 67, 4). Le texte parle toutefois des Perses et de Scythianus comme possibles prédécesseurs du dualisme de Basilide. Scythianus est mentionné dans les Actes d'Archélaüs comme l'autre prédécesseur de Mani; il fut un apprenti de la sagesse égyptienne et pythagoricienne et le premier à inventer le dualisme. Les brèves mentions de Scythianus sont faites par Cyrille de Jérusalem, Hippolyte de Rome et Épiphane de Salamine. Il en résulte que Scythianus fut un maître gnostique alexandrin du $\mathrm{I}^{\mathrm{er}}$ siècle C.E. qui aurait visité l'Inde. Signalons que dans le fragment basilidien des Actes d'Archélaüs figure une phrase par laquelle Basilide semble reconnaître les origines "barbares» de sa doctrine: «Renonçons à cette vaine et curieuse versatilité; examinons plutôt les questions que les barbares eux-mêmes se sont posées, concernant les biens et les maux et les opinions auxquelles ils sont parvenus sur tout cela» $(67,7)$. 
qui «pourrait être le premier, dans la gnose, qui ait posé nettement la question des principes, et qui soit allé chercher les Perses pour justifier sa solution.» ${ }^{12}$

\section{Introduction: La parabole du riche et du pauvre contre le mythe de Poros et Penia}

Le fragment basilidien, cité dans les Actes d'Archélaüs, est introduit par quelques renseignements sur l'activité littéraire de celui qui est présenté comme précurseur du manichéisme. Sa doctrine dualiste est annoncée dès la partie introductive, qui contient déjà une citation de l'écrit de Basilide:

Et tous ses livres contiennent des choses difficiles et très compliquées. Voici le livre treize de ses traités, dont le commencement est ainsi: "À nous, qui sommes en train d'écrire le treizième livre des traités, la parole salvifique (sermo salutaris) apportera les mots nécessaires et abondants - comme par la parabole du riche et du pauvre (Luc 16, 19-31) nous est indiqué d'où pourrait surgir la nature sans racine et sans lieu qui s'étend sur [toutes] les choses». ${ }^{13}$

Hégémonius semble ne connaître les écrits de Basilide que par des morceaux et il se déclare choqué déjà par ces quelques phrases. On y trouve d'abord une expression ambiguë, à savoir «la parole salvifique». Dans le texte latin, qui est une traduction, on lit sermo salutaris, correspondant à l'expression grecque du

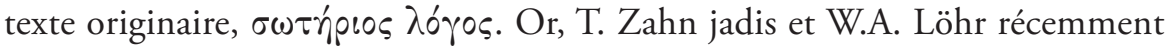
supposent que la meilleure traduction latine aurait été sermo salvatoris, parce qu'elle aurait désigné le texte évangélique utilisé par Basilide comme une collection des logia de Jésus ${ }^{14}$. Cependant, ce n'est pas l'antiquité de l'enseignement qui a choqué Hégémonius, mais l'information selon laquelle la parabole évangélique du riche et du pauvre (voir Luc 16, 19-31) a été interprétée comme réponse à la question «d'où pourrait surgir la nature sans racine et sans lieu qui s'étend

12. S. Pétrement, Le dualisme chez Platon les gnostiques et les manichéens, Paris, PUF, 1947, p. 192.

13. Acta Archelai 67, 5: Et omnes eius libri difficilia quaedam et asperrima continent. Extat tamen tertius decimus liber tractatuum eius, cuius initium tale est: «Tertium decimum nobis tractatuum scribentibus librum necessarium sermonem uberemque salutaris sermo praestabit: per parabolam divitis et pauperis naturam sine radice et sine loco rebus supervenientem unde pullulaverit indicat.» (éd. C.H. Beeson, p. 96; trad. S. Pétrement, Le dualisme, op.cit., pp. 191-192).

14. Cette observation de T. Zahn a été reprise par W.A. Löhr pour montrer que la mention de la parabole sur le riche et le pauvre est une citation des logia de Jésus; cf. Löhr, Basilides und seine Schule, op. cit., pp. 226-227. Ce détail plaide en faveur de l'antiquité de la doctrine de Basilide et donne raison à 'Hippolyte' de Rome qui citait les basilidiens prétendant avoir reçu l'enseignement (l'évangile?) de l'apôtre Matthieu; voir Refut VII, 20, 1. 
sur [toutes] les choses.» Qu'est-ce que donc cette nature - apparemment universelle, parce qu'elle s'étend sur toutes les choses -, mais distinguée par l'expression «sans racine et sans lieu» (sine radice et sine loco)? Or, étant donné

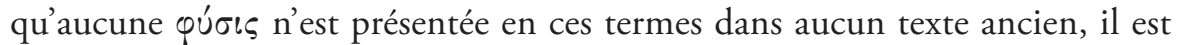
possible que le texte latin donne une traduction approximative de l'original grec; ce qui ne résout pas l'énigme de l'expression basilidienne.

Dans une étude désormais classique, consacrée à l'exégèse des paraboles évangéliques au $\mathrm{II}^{\mathrm{e}}$ siècle, A. Orbe tente d'expliquer ce que pourrait être la nature «sans racine ni lieu» et ses conclusions sont reprises et développées par W.A. Löhr ${ }^{15}$. Celui-ci, par comparaison avec des fragments des écrits de Basilide conservés par Clément d'Alexandrie, démontre que cette expression énigmatique désigne le mal et la souffrance, touchant toutes les choses du monde créé ${ }^{16}$. Dans cette perspective, la péricope évangélique aurait été lue par Basilide comme réponse à la question de l'origine du mal.

Contrairement à ce que pense Hégémonius, il n'est pas aberrant de vouloir établir un lien entre l'origine du mal et la parabole qui raconte les destins croisés d'un homme riche et d'un pauvre qui mendiait près de sa porte. L'étude d'A. Orbe, évoquée plus haut, met en évidence ce rapprochement, élaboré au long d'une lecture qui prend son essor dans le verset de Lc 16, 25, où sont mentionnés les biens ( $\tau \dot{\alpha} \dot{\alpha} \gamma \alpha \theta \dot{\alpha})$ et les maux $\left(\tau \dot{\alpha} \alpha \alpha x \alpha^{\prime}\right)$ reçus pendant la vie, les uns par le riche et les autres par le pauvre ${ }^{17}$. On comprend alors les raisons du choix d'un tel texte évangélique, parce que ni la richesse du riche ni la pauvreté du pauvre ne sont interprétées en termes de récompense ou de punition. Puis, le renversement de leurs situations respectives après la mort n'est pas assorti d'un jugement moral. Ces détails auraient sans doute suffi à Basilide pour établir un parallèle entre le bien et le mal, fondé sur les éléments présents dans le texte évangélique, et pour s'en servir à introduire sa doctrine dualiste. Cette supposition semble trouver sa confirmation plus loin dans le texte basilidien cité par Hégémonius:

15. A. Orbe propose deux réponses: (1) la «nature» est l'ensemble des êtres, en tant que issus du mélange de la lumière et des ténèbres; (2) la «nature» désigne le mal et la souffrance dans le monde. Il penche pour la seconde solution, adoptée également par W.A. Löhr; voir A. Orbe, Parábolas evangélicas en San Ireneo, vol. 2, Madrid, 1972, pp. 328-332; W.A. Löhr, Basilides und seine Schule, op. cit., pp. 227-229.

16. Il s'agit principalement du Fragment 9 (Strom IV, 88, 5) qui est pourtant inséparable d'un débat plus vaste, concernant le martyre comme une rétribution sous forme de punition une idée basilidienne que Clément combat surtout dans le Stromate IV 81,1-83,1. Pour l'analyse de ce débat, voir A. Le Boulluec, La notion d'hérésie dans la littérature grecque, $I I^{e}-I I I^{e}$ siècles, vol. 2, Clément d'Alexandrie et Origène, Paris, Études Augustiniennes, 1985, pp. 304-312. Voir aussi Löhr, Basilides und seine Schule, op. cit., pp. 122-159.

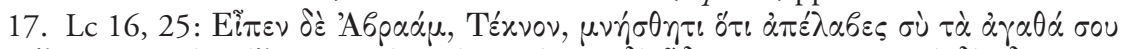

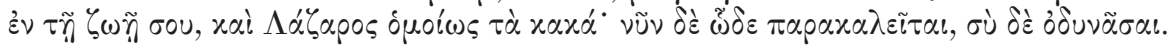
(texte grec éd. Nestle-Aland). 
Car il en est qui ont dit que les principes de toutes choses sont au nombre de deux, auxquels les biens et les maux se rattachent, et que ces principes eux-mêmes sont sans principe et inengendrés. C'est-à-dire qu'il y a eu au commencement la lumière et les ténèbres, qui n'étaient issues que d'elles-mêmes et de rien d'autre. Quand chacun de ces principes était en lui-même, chacun menait sa vie propre, celle qu'il voulait et telle qu'il lui convenait. ${ }^{18}$

Le passage cité mentionne «les biens et les maux» qui pourraient renvoyer au verset de Lc 16, 25, ce qui permet le passage vers l'exposé doctrinal plus élaboré. En fait, Basilide se prépare à exposer l'essentiel de sa doctrine en présentant d'abord un couple de contraires, formé de la lumière et des ténèbres, antérieur à l'opposition entre les biens et les maux. Ayant préalablement affirmé que le mal, en tant que "nature sans racine ni lieu», fait partie constitutive du monde, Basilide peut se servir du texte évangélique pour montrer que les maux et les biens ne sont pas d'ordre moral mais métaphysique. En tant que principes contraires, la lumière et les ténèbres imitent le rapport qui existe entre le riche et le pauvre de l'évangile. Cependant, avant de passer à cette partie de l'enseignement de Basilide, arrêtons-nous sur l'arrière-plan platonicien de son raisonnement.

Dans sa reconstruction de l'exégèse basilidienne, A. Orbe a signalé la ressemblance entre la parabole évangélique du riche et du pauvre et le mythe platonicien de Poros et Pénia (Banquet 203c-e). Les liens entre ces deux textes seraient à établir à partir des versets suivants: «Un pauvre nommé Lazare, gisait près de son portail couvert d'ulcères. Il aurait désiré se rassasier de ce qui tombait de la table du riche...» (Lc 16, 20-21) ${ }^{19}$. Le passage cité ouvre la possibilité d'interpréter les personnages du pauvre et du riche en les comparant avec Pénia la pauvre et Poros le riche. Suivant cette comparaison, A. Orbe repère chez Basilide une lecture platonisante de la péricope évangélique et il la fait apparaître à partir de plusieurs rapprochements textuels ${ }^{20}$. Outre la ressemblance entre les personnages, il y a une ressemblance de contexte dans lequel les protagonistes des deux textes se rencontrent. Il y a d'abord le banquet - l'un

18. Acta Archelai 67, 7-12: Quidam enim horum dixerunt initia omnium duo esse, quibus bona et mala adsociaverunt, ipsa dicentes initia sine initio esse et ingenita; id est in principiis lucem fuisse ac tenebras, quae ex semet ipsis erant, non quae <genitae $>$ esse dicebantur. 8. Haec cum apud semet ipsa essent, proprium unumquodque eorum vitam agebant quam vellent et quale sibi conpeteret; omnibus enim amicum est quod est proprium et nihil sibi ipsum malum videtur. (éd. C.H. Beeson, p. 96; trad. S. Pétrement, Le dualisme, op.cit., pp. 191-192).

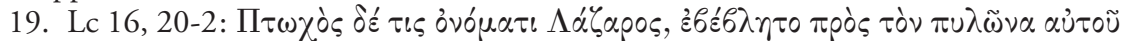

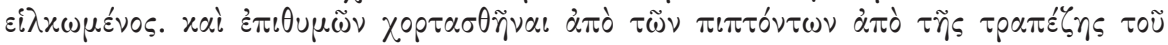
$\pi \lambda$ ou ${ }^{\prime}$ iou. (éd. Nestle-Aland).

20. Voir A. Orbe, Parábolas evangélicas en San Ireneo, op. cit., pp. 328-332; W.A. Löhr, Basilides, op. cit., pp. 226-227. 
se tient dans le jardin de Zeus, l'autre à la maison du riche. Dans le texte de Platon, Pénia «se tint auprès de la porte» (203b5), tout comme Lazare de l'évangile. Mais avant tout, il y a un manque et un désir de l'assouvir qui poussent Pénia vers Poros et le pauvre vers la table du riche. Le désir va réapparaitre encore dans le texte de Basilide, explicitement évoqué comme moteur des relations entre la lumière et les ténèbres. Il est donc possible de traiter la lecture platonisante de la parabole comme une sorte d'introduction à l'exposé sur le couple de principes contraires; un couple à la fois uni et séparé par le désir.

La recherche des éléments qui annoncent l'apparition de la doctrine des principes contraires amène à reconsidérer la mention de «nature sans racine et sans lieu». Notre but n'est pas de remettre en cause son identification avec le mal - étant donné l'importance de cette question chez Basilide - mais de montrer qu'elle s'inscrit dans l'introduction à la doctrine de deux principes. Ayant constaté l'absence des mentions de la nature «sans racine et sans lieu» dans la littérature philosophique, l'attention des chercheurs s'est focalisée sur une expression semblable, utilisée par les disciples de Simon le Mage dans l'Apophasis Megalè. La comparaison entre le fragment de l'enseignement des simoniens et celui de Basilide se montre révélatrice. Or, dans l'Apophasis est racontée l'origine de deux éons - Dynamis et Epinoia - lesquels, en tant que deux éléments, masculin et féminin, forment l'unique principe androgyne; ils sont appelés «rejetons qui n'ont ni commencement ni fin, issus d'une seule et même racine» ${ }^{21}$. Il faut rappeler que le même motif, à savoir deux principes "auxquels s'attachent les biens et les maux», se trouve dans la citation de Basilide. Si on compare les descriptions de ces couples de principes, on repère

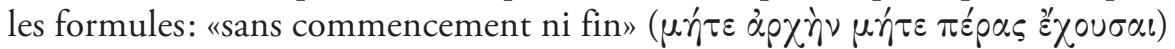
chez les simoniens et "principes sans principe et inengendrés» (initia sine initio esse et ingenita) chez Basilide. Cette ressemblance permet d'interpréter la formule «sans racine et sans lieu» (sine radice et sine loco) au même sens que «sans commencement et sans fin"; cela est d'autant plus plausible qu'une opposition entre le masculin et le féminin est présente chez Simon et chez Basilide.

La comparaison entre ces deux doctrines s'avère utile, car elles possèdent en commun la conception dualiste du terme àp $\chi \hat{\eta}$. Et si chez les simoniens il s'agit d'un principe double, ayant en lui les éléments contraires, masculin et féminin, chez Basilide il est question de deux principes contraires, séparés l'un de l'autre. La radicalité des positions de Basilide se manifeste aussi dans l'ampleur de son projet qui est à la fois métaphysique et cosmogonique.

21. Réfutation VI, 18, 2-4; trad. française dans J.M.A. Salles-Dabadie, Recherches sur Simon le Mage, I: L’ «Apophasis megalè», Paris, J. Gabalda, 1969, pp. 36-37. La ressemblance entre le fragment de Simon le Mage et celui de Basilide est analysée par A. Orbe, Parábolas evangélicas, op. cit., pp. 329-330. 


\section{Les principes contraires - le bien et le mal / la lumière et les ténèbres}

Le fragment basilidien cité par Hégémonius se distingue par sa façon romanesque de présenter l'origine des choses. Nous insistons sur le fait que ce passage n'est pas seulement cosmogonique - comme l'interprète Löhr - mais que Basilide parle de principes métaphysiques, tout en leur attribuant les noms des éléments physiques. Sa méthode ressemble à l'analogie du soleil, dont Platon se sert pour répondre à la question concernant la nature de l'idée du Bien. Si on soupçonne Basilide d'adopter la même stratégie, cela est rendu possible par certains regroupements de termes, à savoir que «les biens et les maux se rattachent» à ceux qu'il appelle la lumière et les ténèbres, ou encore que la lumière soit "la meilleure chose». Le rapport avec la cosmogonie est toutefois perceptible, même si Basilide ne traite pas explicitement de l'origine du monde: au moins pas avant de mentionner la matière à la fin du récit. Il serait donc plus exact de dire qu'il offre une explication de la présence du mal dans le monde, en mettant en scène les ténèbres et la lumière. Cette visée est annoncée dans la conclusion qui annonce l'absence du bien parfait dans le monde, parce que la seule forme du bien proviendrait du reflet de la lumière sur la matière du monde où vivent les hommes.

Mais avant d'arriver là, il nous faut bien comprendre l'articulation entre deux couples de principes contradictoires - le bien et le mal d'un côté, la lumière et les ténèbres de l'autre. En procédant par recoupement des connotations liées à ces deux types d'opposition, Basilide réussit à éviter les connotations morales du bien et du mal, au moins dans un premier temps. Toutefois, les notions de bien et de mal restent sous-jacentes à l'opposition entre la lumière et les ténèbres, en leur donnant une dimension métaphysique. C'est ainsi que la lumière devient un être Bon et les ténèbres un être Mauvais. Basilide décrit les rapports entre eux en procédant par étapes, mais, avant que le tout commence, il affirme que les principes furent séparés par une ignorance réciproque:

Quand chacun de ces principes était en lui-même, chacun menait sa vie propre, celle qu'il voulait et telle qu'il lui convenait. Car à toute chose est cher ce que lui est propre et rien ne paraît mauvais à soi-même. ${ }^{22}$

Le texte nous dit qu'à ce stade, les principes, étant séparés l'un de l'autre, ne se définissent pas en tant que «bien» ou «mal», ni par aucun autre nom.

22. Acta Archelai 67, 8: Haec cum apud semet ipsa essent, proprium unumquodque eorum vitam agebant quam vellent et quale sibi conpeteret; omnibus enim amicum est quod est proprium et nihil sibi ipsum malum videtur. (éd. C.H. Beeson, p. 96; trad. S. Pétrement, Le dualisme, op. cit., pp. 191-192). 
Basilide postule donc l'existence de deux principes fondamentaux, irréductiblement distincts et demeurant dans un état d'équilibre. La situation bascule au moment où ils apprennent chacun l'existence de l'autre:

Mais après que chacun [= principe] fut parvenu à la connaissance de l'autre, et que les ténèbres eurent contemplé la lumière, comme une chose meilleure, elles en eurent le désir, et elles poursuivirent la lumière, désirant s’y joindre et y participer. Telle était donc l'action des ténèbres.

Pour la lumière, elle n'acceptait rien des ténèbres, ni ne venait à les désirer, si ce n'est qu'elle éprouva, elle aussi, le désir de les regarder.

Elle les regarda donc, comme au moyen d'un miroir. Ainsi un reflet (enfasis), c'est-à-dire quelque couleur de lumière, se fit seule sur les ténèbres; mais la lumière elle-même regarda seulement et s'éloigna, n'ayant pris aucune parcelle des ténèbres. Les ténèbres au contraire, saisirent le regard de la lumière et le reflet ou la couleur que la matière (yles enfasin vel colorem) avait reçue, dans cet instant où elles lui avaient fait horreur. ${ }^{23}$

Nous avons divisé le texte en trois parties pour mettre en évidence les étapes successives des rapports entre les deux principes. Ainsi, après l'état originaire d'immobilité et d'ignorance, il y a trois étapes, dont chacune correspond à une certaine activité d'un ou de deux principes: (1) activité des ténèbres contemplation de la lumière et le désir de la suivre, (2) activité de la lumière refus d'être affectée par les ténèbres et le désir de les voir, (3) les conséquences pour les deux - le regard de la lumière à travers le miroir donne aux ténèbres un reflet de lumière, mais provoque aussi la séparation ultime des deux principes.

Le drame commence avec la rencontre qui équivaut à la connaissance de l'un par l'autre. Basilide parle même de la «contemplation» dans laquelle les ténèbres apprennent à connaître la lumière en la reconnaissant en tant qu'une "chose meilleure» (res melior). Ainsi l'équilibre initial est rompu par la connaissance venant de cette contemplation. La définition d'un des principes comme une

23. Acta Archelai 67, 7-12: Quidam enim horum dixerunt initia omnium duo esse, quibus bona et mala adsociaverunt, ipsa dicentes initia sine initio esse et ingenita; id est in principiis lucem fuisse ac tenebras, quae ex semet ipsis erant, non quae <genitae > esse dicebantur. Haec cum apud semet ipsa essent, proprium unumquodque eorum vitam agebant quam vellent et quale sibi conpeteret; omnibus enim amicum est quod est proprium et nihil sibi ipsum malum videtur. Postquam autem ad alterutrum agnitionem uterque pervenit et tenebrae contemplatae sunt lucem, tamquam melioris rei sumpta concupiscentia insectabantur ea et coadmisceri ac participari de ea cupiebant. Et tenebrae quidem haec agebant, lux vero nequaquam ex tenebris quicquam recipiebat in sese nec in earum desiderium veniebat, tantummodo quod etiam ipsa spectandi libidinem passa est. Et quidem et respexit eas velut per speculum. Enfasis igitur, id est color quidam lucis, ad tenebras factus est solus, sed lux ipsa respexit tantummodo et abscessit, nulla scilicet parte sumpta de tenebris. Tenebrae vero ex luce sumpserunt intuitum et yles enfasin vel colorem, in quo ei displicuerant. (éd. C.H. Beeson, p. 96; trad. S. Pétrement, Le dualisme, op. cit., pp. 191-192). 
«chose meilleure» est fondamentale, parce qu'il ne s'agit pas d'une gradation les principes basilidiens sont des contraires, pas des semblables qui se distinguent qualitativement l'un de l'autre. Il faut donc interpréter res melior au sens absolu: la lumière se fait connaître comme le meilleur des êtres, le bien en soi. En conséquence de cette découverte, les principes contraires se positionnent respectivement comme «le bien en soi» et son contraire, à savoir «le mal en soi». Ce moment coïncide avec la naissance du mal en tant que principe métaphysique.

Déjà ces premières lignes trahissent une inspiration platonicienne, plutôt superficielle mais certaine. La suite du texte ne fait que renforcer cette impression, dans la mesure où elle s'inspire des livres VI et VII la République. Le plus significatif serait le passage 505a-509b, où Platon parle de la connaissance du Bien, puis explique sa nature en utilisant l'analogie du soleil. Il est également possible d'interroger l'analogie de la caverne, parce qu'elle permet de faire le lien entre la connaissance et l'expérience. Quant à la contemplation comme moyen de connaissance, ce thème est inspiré par le mythe du voyage des âmes et leur contemplation des Idées (Phèdre $247 \mathrm{c}-\mathrm{e}$ ). Cependant, quel que soit le texte qui a inspiré Basilide, il est évident que Platon n’oppose au Bien aucune idée du mal, parce que ce dernier est une forme d'ignorance et de mal $\operatorname{agir}^{24}$. C'est là où se situe l'originalité de l'interprétation basilidienne du principe du Bien.

Dès que les ténèbres reconnaissent la lumière comme le Bien absolu, elles se mettent à la désirer. Dans le texte latin figure le substantif concupiscentia, fréquent chez les auteurs chrétiens, qui fait penser au désir amoureux, d'autant plus qu'il est suivi du verbe cupere pour compléter la description. Mais la comparaison avec les textes platoniciens montre - une fois de plus - que la traduction latine prête à confusion. Étant donné que Basilide s'est également inspiré du mythe de Poros et Pénia du Banquet, il est fort possible que cette inspiration continue à se manifester dans sa conception du rapport entre le bien et le mal. En quoi alors les relations entre la lumière et les ténèbres ressemblent-elles à celles entre Pénia et Poros du Banquet 203b-c? Tout d'abord, dans le texte platonicien il n'est pas question du désir amoureux; Pénia s'approche de Poros poussée par sa pénurie (à $\pi \circ p i ́ \alpha, 203 b 6)$ et Platon fait un jeu de mots sur Пópos,

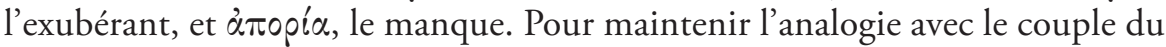
Banquet, il faut remplacer le désir amoureux par une autre forme du désir. Quand on cherche un terme pour décrire la réaction provoquée chez les ténèbres par la

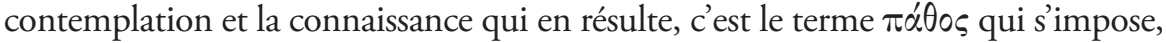
puisqu'il désigne à la fois l'affection et la passion. Tant que ce terme est généralement utilisé pour exprimer la passion subie ou suscitée, on devrait le traduire plutôt par «affection», bien que le sens «passion» ou «désir» soit tout aussi possible. Ainsi, traduire $\pi \dot{\theta} \theta \circ$ sar concupiscence réduit son champ sémantique.

24. Pour l'étude des structures dualistes chez Platon et la place du mal, voir S. Pétrement, Le dualisme, op. cit., pp. 79-85. Sur le Bien en tant que limité par la nécessité, idem, pp. 39-44. 
Voici comment Platon dans le Sophiste envisage le lien entre la connaissance et l'affection, dans le cadre d'un long exposé sur l'être, qui aboutit dans la

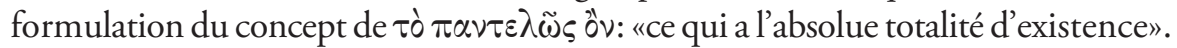
Alors qu'il présente les différentes doctrines - les «mythes» - anciennes, l'Étranger annonce: "Celui qui affirme que l'être est soit un couple, soit seulement une unité, se trouve acheminé vers des milliers d'autres impasses infranchissables» ${ }^{25}$. Pour surmonter les impasses en question, l'Étranger propose de trouver l'unité entre les deux éléments qui forment un couple de l'être - "qu'énoncez-vous sur les deux lorsque vous dites que les deux et que chacun, sont?» (Sophiste 243e). Basilide laisse cette question sans réponse et échafaude un système dans lequel l'être est un couple de contraires; il s'écarte ainsi de la conception platonicienne de l'être. Ce qui rattache toutefois cette doctrine à celle de Basilide réside dans la manière de présenter la communication, au terme de laquelle l'âme apprend à connaître l'être. L'Étranger dit:

Si connaître c'est faire quelque chose, il s'ensuivra nécessairement que ce qui est connu pâtisse. Et selon le même raisonnement, la réalité existante sera connue par la connaissance, et, dans la mesure où elle ne peut que pâtir lorsqu'elle est connue, elle sera donc mue; ceci, en effet, ne peut pas arriver à ce qui est en repos. ${ }^{26}$

La connaissance, en tant qu'action, affecte l'objet et le sujet. Il est frappant que Basilide emprunte la logique platonicienne, selon laquelle le $\pi \dot{\theta} \theta 0 \varsigma$ concerne les deux agents, celui qui connaît et l'autre qui se fait connaître: autrement, l'un des deux serait resté dans son immobilité primitive. Maintenant on comprend pourquoi la lumière ne continue pas à garder son immobilité, en se laissant contempler et désirer mais, affectée par l'action des ténèbres, elle expérimente un $\pi \alpha ́ \theta 0 \varsigma$ à son tour. On garde néanmoins l'impression que l'inspiration platonicienne chez Basilide est sélective et soumise à quelques relectures.

Si dans le Sophiste Platon établit le lien entre la connaissance et l'affection, que dit-il au sujet de la contemplation - est-elle également capable de susciter des passions, voire des affections? Le seul endroit où Platon associe ces deux termes est un passage de la République qui traite des affections de l'âme provoquées par la «vue» des œuvres des poètes (606a-b). Ce passage oriente la réflexion philosophique postérieure, qui a pris l'habitude d'aborder le problème des passions suscitées par la contemplation dans le cadre de la réflexion sur l'âme et ses mouvements, tenant toutefois pour acquise l'opinion que les

25. Sophiste 245d-e1; trad. N. Cordero, GF Flammarion, Paris, 1993, p. 147. Pour le commentaire sur l'expression $\tau \grave{c} \pi \alpha \nu \tau \varepsilon \lambda \omega \tilde{\omega} \varsigma$ ôv, traduit comme «l'être total», voir ibidem, p. 250

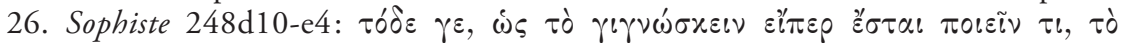

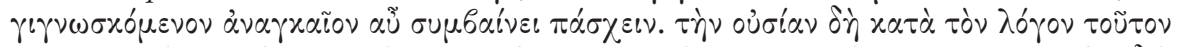

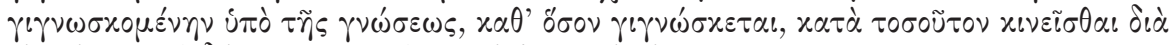

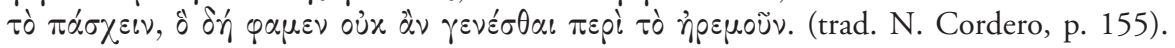
Voir aussi le commentaire, pp. 250-251. 
intelligibles (ou les incorporels) ne sont pas atteints par les passions, contrairement à la matière ${ }^{27}$. Tout cela fait penser que les rapports entre la lumière et les ténèbres chez Basilide sont fondés, de manière générale, sur l'opposition entre l'intelligible et le sensible. Or, cette distinction, fondamentale dans le platonisme, se décline en plusieurs types de relations: entre l'esprit et la matière, entre le corps et l'âme. Et Basilide semble réduire plusieurs oppositions à une seule.

L'exemple d'une telle réduction arrive avec la description de l'activité de la lumière. Tout ce que Basilide a dit sur elle jusque-là prouve qu'elle représente le meilleur des intelligibles, le principe du Bien, l'objet de contemplation et de désir. Or, cette lumière est atteinte par le $\pi \alpha ́ \theta 0 \varsigma$. Son activité, certes, n'est pas symétrique à celle des ténèbres, mais elle reste néanmoins affectée par le fait d'être contemplée et désirée ${ }^{28}$. Ici, on constate que le raisonnement de Basilide suit son propre chemin, puisqu'il soumet la lumière aux passions contradictoires: d'un côté, elle n'accepte rien des ténèbres et n'est pas affectée par elles (nec in earum desiderium veniebat), mais, en même temps, elle éprouve le désir de les regarder (spectandi libidinem) dans un miroir. Les termes latins donnent toujours au texte l'allure d'une histoire d'amour, ce qui ne devrait pas cacher les références platoniciennes de la pensée de Basilide.

Dans la perspective platonicienne, la première réaction de la lumière n'a rien d'étonnant: en tant qu'elle représente l'intelligible, sinon le Bien en soi, elle n'a pas à subir une quelconque affection. Cette première attitude de la lumière devient plus compréhensible après la lecture du livre VI de la République, et plus précisément après les explications sur la possibilité de connaître le Bien.

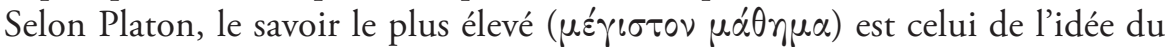
Bien (505a), parce que grâce à cette idée il est possible de reconnaître les biens véritables au milieu des choses qui seulement semblent être bonnes (505c-d). Cependant, lorsque les interlocuteurs de Socrate le pressent d'expliquer la nature du Bien, il esquive la réponse et leur propose trois analogies, celles du soleil, de la ligne et de la caverne. Le soleil, source de la lumière du jour, permet de voir les objets colorés, contrairement à l'obscurité nocturne qui rend leur claire vision impossible. Par analogie au soleil, l'idée du Bien possède la faculté de rendre les choses «visibles», au sens où elle est la cause de la connaissance et de la vérité (508e-509a) - tout en restant aussi l'objet de la connaissance. En tant

27. Telle est la position «classique», représentée par Aristote (De l'âme I, De gen. I) et partagée par les platoniciens - tel que Plutarque dans Sur Isis et Osiris - qui ont interprété les passages du Timée (48e-50a, sur père et mère) et du Banquet (203c-e, sur Poros et Pénia) dans le même sens. Les thèses de Plotin sur la matière intelligible et sur l'unité de l'âme vont à l'encontre de ces positions; cf. le traité 26 (III, 6) Sur l'impassibilité des incorporels ainsi que les traités 27-29 (IV, 3-5), Sur les difficultés relatives à l'âme. Le traité 30 (III, 8) Sur la contemplation défend la thèse que tous les êtres contemplent l'Un.

28. W.A. Löhr remarque cette inégalité entre les activités de deux principes, mais la conclusion qu'il en tire lui fait parler d'un certain adoucissement du dualisme; voir Basilides und seine Schule, op. cit., pp. 247-249. 
qu'objet de connaissance, le Bien possède une beauté extraordinaire qui surpasse toute connaissance et toute vérité (509a). Socrate en tire la conclusion suivante:

Je pense que tu admettras que le soleil confère aux choses visibles non seulement le pouvoir d'être vues, mais encore la genèse, la croissance et la subsistance, encore que lui-même ne soit aucunement genèse. ${ }^{29}$

L'idée du Bien confère aux choses la cognoscibilité, leur être et leur essence, mais ce Bien n'est pas l'essence, mais «quelque chose au-delà de l'essence», हैं

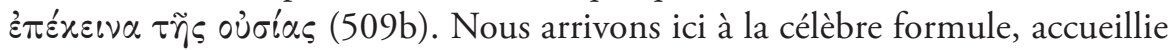
par cet éclat de rire de Glaucon: «Quelle merveilleuse transcendance!» Indépendamment du sens technique, acquis par cette formule ultérieurement, il est clair que l'idée du Bien surpasse en beauté ce dont elle est cause, à savoir la

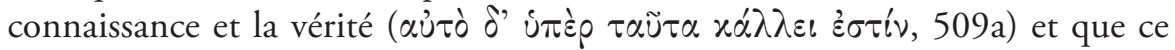
dépassement consiste dans le fait de ne pas partager la même essence, voire ne

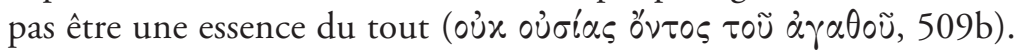

Les caractéristiques de l'idée du Bien, que nous venons de présenter sommairement, sont suffisantes pour expliquer l'action de la lumière dans le texte de Basilide. Notre lecture qui voit dans la lumière une interprétation basilidienne du principe du Bien, permet d'expliquer pourquoi la lumière, dans un premier temps, n'est pas affectée par les ténèbres et ne partage pas leur essence. Cela veut dire que c'est la seconde réaction de la lumière qui est surprenante. À vrai dire, pour Platon, la question: comment le Bien - si on prend l'analogie du soleil - est-il atteint (et s'il l'est!) par son action d'éclairer les choses, n'aurait aucun sens... Et pourtant, Basilide semble y répondre, parce que son Bien lumière finit par éprouver le désir de voir les ténèbres dans le miroir. Il est donc difficile de lui attribuer le statut métaphysique «au-delà de l'essence», étant donné qu'elle est affectée par l'action des ténèbres et qu'elle suit cette affection. Et pourtant, Basilide semble lui accorder toujours ce statut-là.

Ici commence la troisième étape des rapports entre les deux principes basilidiens: en conséquence de l'affection réciproque, subie par la lumière et les ténèbres, une sorte de rencontre entre les deux se produit. Le contact reste néanmoins limité et se déroule dans des conditions particulières, puisque la lumière ne regarde pas les ténèbres directement, mais à travers un miroir. Son regard est médiatisé, ce qui le rend différent de celui que les ténèbres lui ont adressé en premier. Au passage, signalons l'absence d'un quelconque organe visuel par lequel les ténèbres et la lumière puissent se regarder - Basilide n'en dit pas un mot ${ }^{30}$. En

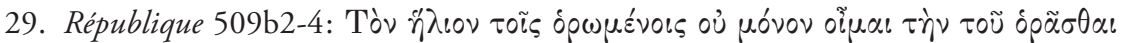

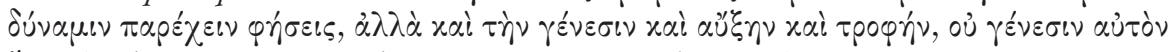

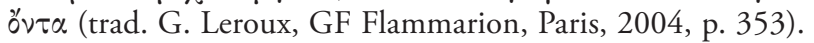

30. On pense à la notion de "œil de l'âme» ou une autre faculté mentale permettant la vision. Sur ce sujet voir l'étude d'A. Vasiliu, «La parabole du regard», dans Dire et voir. 
remplaçant une vision directe par un reflet dans le miroir, Basilide souligne encore l'absence de communication entre les deux principes; ils n'ont rien en partage, ils ne se «touchent» même pas par la vue ${ }^{31}$. Voici encore une autre façon d'exprimer la radicale séparation entre la lumière et les ténèbres, entre le bien et le mal - ou plutôt une tentative de préserver cette séparation.

Il arrive cependant un moment où cette séparation semble ne plus fonctionner le regard de la lumière touche les ténèbres. On peut encore sauver la pensée de Basilide, en rappelant l'analogie platonicienne du soleil qui éclaire les choses, en leur donnant la coloration, sans être atteint par elles en retour (voir République $507 \mathrm{~d}-\mathrm{e})$. De manière semblable, seul le regard de la lumière se pose sur les ténèbres comme "un reflet, à savoir une certaine couleur de la lumière» (enfasis igitur, id est color quidam lucis). À cet endroit, le traducteur latin a rencontré une difficulté terminologique qu'il a décidé de contourner par la translittération

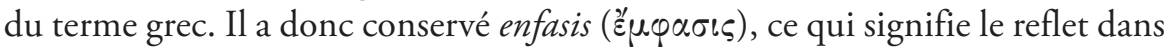
un miroir ou l'apparence. Il n'y a aucun doute concernant l'utilisation de ce terme par Basilide parce que, dans le texte qui suit, il apparait encore deux fois, et toujours en translittération ${ }^{32}$. En outre, Basilide le fait toujours suivre d'une curieuse explication: «reflet» veut dire "couleur».

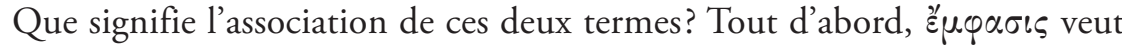
dire exactement le reflet, le scintillement du miroir ou de la surface de l'eau. Ce terme est utilisé par Aristote dans le traité De sensu pour distinguer entre la vue et l'apparence de la chose. Alexandre d'Aphrodise, dans son commentaire sur ce traité aristotélicien, remarque que la vue consiste à exercer une puissance qui s'actualise lorsqu'il y a une apparence $(\text { (ै } \mu \varphi \alpha \sigma \iota \varsigma)^{33}$. Comme le montre A. Vasiliu dans une étude consacrée à la vue selon Plotin et selon Aristote, une des différences entre ces deux approches consiste dans le fait que la doctrine de Plotin est psychologique, tandis que celle d'Aristote est physique - et

La parole visible du Sophiste, Paris, Vrin, 2008, pp. 301-357. Sur les éléments constitutifs de l'acte de contemplation dans la tradition platonicienne; voir A. Vasiliu, «La contemplation ou le lien invisible selon Plutarque et Plotin", dans Images de soi dans l'Antiquité tardive, Paris, Vrin, 2012, pp. 105-147.

31. Voir A. Vasiliu, "Toucher par la vue. Plotin en dialogue avec Aristote sur la sensorialité de l'incorporel», Les Études philosophiques, 4 (2015), pp. 555-580.

32. Acta Achelai 67, 10: "Les ténèbres au contraire, saisirent le regard de la lumière et le reflet ou la couleur que la matière avait reçue, dans cet instant où elles lui avaient fait horreur. Comme les plus méchants avaient pris du meilleur, non la vraie lumière, mais une certaine apparence de celle-ci et un reflet, ils en tirèrent à eux quelque bien par un rapt qui en changeait la nature.» Tenebrae vero ex luce sumpserunt intuitum et yles enfasin vel colorem, in quo ei displicuerant. Cum ergo nequiores de meliore sumpsissent non veram lucem, sed speciem quandam lucis atque enfasin, ... boni raptiva mutatione traxerunt. (éd. C.H. Beeson, p. 96; trad. S. Pétrement, Le dualisme, op. cit., pp. 191-192).

33. Cette distinction est faite à l'occasion de la critique des théories de Démocrite; voir De sensu 438a6-12; voir A. Vasiliu, «Toucher par la vue», art. cit., pp. 573-575. 
pourtant, Plotin poursuit un dialogue avec la pensée aristotélicienne exprimée dans les traités naturels ${ }^{34}$. Signalons néanmoins que ce vocabulaire aristotélicien n'est pas ignoré des platoniciens, et que même chez Plotin il y a une seule

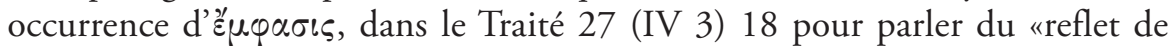
l'Intellect» dans l'âme ${ }^{35}$. Cependant, le terme plotinien habituel pour parler de la «trace de l'intellect» sur l'âme (Traité 28 [IV 4] 18) ou la "trace de l'être» laissé par l'Un (Traité 30 [III 8], 11) est ''́vos - un reflet qui demeure empreint

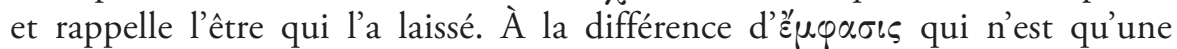
apparence scintillante, 'í $\chi$ vos est une empreinte du pied. Eu égard aux efforts déployés par Basilide pour maintenir la séparation entre la lumière et les ténèbres, les raisons de son choix de vocabulaire sont évidentes.

Et maintenant, que veut-il dire par la "couleur», terme employé comme quasi synonyme d'é $\mu \varphi \propto \sigma \iota \varsigma$ ? La solution est à chercher chez Aristote, dans sa

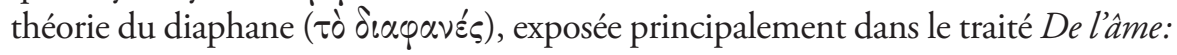

La lumière est l'acte de cette substance, du diaphane, en tant que diaphane, et là où le diaphane est présent seulement en puissance, là aussi existe l'obscurité. La lumière, elle, est comme la couleur du diaphane, quand le diaphane est réalisé en entéléchie sous l'action du feu ou de quelque chose qui ressemble au corps situé dans la région supérieure, car à cette dernière substance appartient aussi un attribut qui est un et identique avec le feu. Nous venons ainsi d'indiquer que la nature du diaphane et celle de la lumière ne sont ni du feu ni, en général, un corps, ni un effluve d'aucun corps (...), mais qu'elle est, en réalité la présence du feu ou de quelque chose de ce genre dans le diaphane. ${ }^{36}$

Cet aperçu de la théorie aristotélicienne du diaphane facilite la lecture des termes utilisés dans le texte basilidien. En effet, le diaphane est une certaine couleur de la lumière, ou plutôt un medium transparent, un milieu entre les

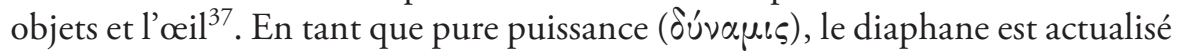
par la lumière; mais, en absence de celle-ci, il est obscurité. Même reprise de manière superficielle, la notion du diaphane s'applique bien aux idées basilidiennes; en fait, elle met en évidence l'opposition radicale entre les principes, en tant qu'agissant sur le diaphane de manière radicalement différente: le reflet

34. Voir A. Vasiliu, ibid., pp. 565-566; 575-576.

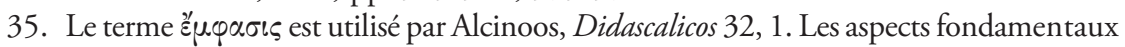
de la théorie platonicienne de la vision sont traités par A. Vasiliu dans le chapitre «La contemplation ou le lien invisible selon Plutarque et Plotin», op. cit., pp. 105-147.

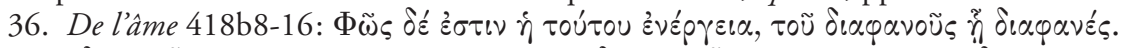

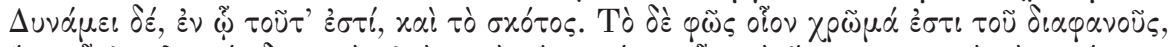

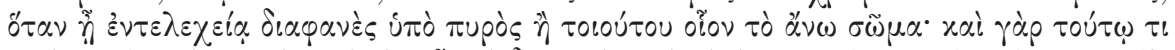

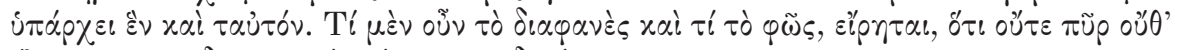

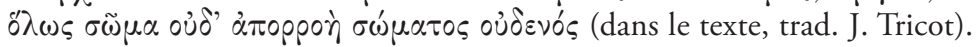

37. Voir A. Vasiliu, Du diaphane. Image, milieu, lumière dans la pensée antique et médiévale, Paris, Vrin, 1997. 
de la lumière suscite en lui la couleur, tandis que les ténèbres le rendent invisible. Cela fait partie d'une longue série d'efforts de Basilide pour préserver l'opposition entre les principes. Le diaphane est la couleur actualisée par le regard de la lumière, un reflet qui n'est pas une parcelle de la lumière elle-même, mais qui provient de son regard transmis par le miroir... Une fois de plus, Basilide cherche à éloigner les principes et à atténuer l'affection de l'un sur l'autre. Mais cette tentative rencontre des difficultés considérables. La réaction de la lumière, à qui le seul aperçu des ténèbres fait horreur et qui s'enfuit pour ne pas en recevoir la moindre parcelle, exprime, certes, une opposition radicale, mais également un lien entre les deux principes qui s'affectent mutuellement. L'horreur et la fuite de la lumière sont proportionnelles à l'admiration et au désir des ténèbres. On pourrait parler d'un «effet miroir», d'une symétrie inversée qui définit les rapports entre la lumière et les ténèbres.

Il reste encore une dernière conséquence de l'action de la lumière. Les

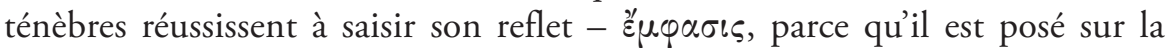
matière. Cette affirmation aussi n'a du sens qu'en rapport avec la théorie aristotélicienne du diaphane. Étant donné que dans l'obscurité le diaphane n'est qu'une pure potentialité sans couleur, Basilide ne peut pas prétendre que les ténèbres puissent retenir une "couleur de la lumière». Contrairement aux ténèbres, dont la nature est privation de la lumière et de la couleur, la nature du diaphane est définie positivement: elle est commune à l'air et l'eau, et c'est pourquoi le diaphane pénètre tous les corps composés qui renferment ces deux éléments (voir De l'âme 418b4-8). Par conséquent, Basilide peut dire que c'est la matière qui a reçu le reflet ou la couleur. Le rapport entre la matière et les ténèbres n'est pas défini, mais il n'y a pas de raison de remettre en question l'identification entre ces deux êtres. Les ténèbres chez Basilide peuvent être le synonyme d'une matière première, sans qualités, en quoi il ne se distinguerait pas des auteurs chrétiens du $\mathrm{II}^{\mathrm{e}}$ siècle qui partagent avec les Grecs l'idée de l'éternité de la matière ${ }^{38}$. Certes, la manière dont Basilide l'affirme nous paraît abrupte; néanmoins cela est assez clair, dès les premières lignes: dans son texte les ténèbres représentent le vaste domaine du sensible.

L'identification des ténèbres à la matière trouve sa justification dans la dernière partie de notre passage, qui révèle sans doute l'objectif de la démonstration faite par Basilide:

Comme les plus méchants avaient pris du meilleur, non la vraie lumière, mais une certaine apparence de celle-ci et un reflet, ils en tirèrent à eux quelque bien par un rapt qui en changeait la nature. De là vient qu'il n'y a point de bien parfait en ce

38. Voir Justin Martyr, Apologie I 10, 2-3; 59, 1. 5; Tatien, Discours aux Grecs 4, 1-2; $12,1-11$. Sur les conceptions chrétiennes de la création à partir de la matière sans qualités, voir I. Jurasz, «Dieu comme dêmiourgos et poiêtês des auteurs chrétiens du II siècle», Chôra, 13 (2015), pp. 217-249. 
monde, et que le bien qui s'y trouve est très faible, parce que faible fut déjà celui qui a été conçu au commencement. Cependant grâce à ce peu de lumière, ou plutôt grâce à cette apparence de lumière, les créatures ont eu la force d'engendrer une ressemblance de ce mélange de lumière qu'elles avaient reçu. Et c'est là cette créature que nous voyons. ${ }^{39}$

Voici le véritable sujet de Basilide: le bien dans le monde, ou plutôt une apparence du bien. Les méchants (est-ce qu'il y a des bons parmi les hommes?) peuvent ravir une apparence, un reflet seulement, de ce bien suprême, mais au prix d'en changer la nature. Ce qu'ils appellent «bien» n'est qu'une apparence de l'apparence du bien véritable. Basilide le dit explicitement: «il n'y a point de bien parfait en ce monde» (nec perfectum bonum est in hoc mundo), ce qu'il faut entendre comme absence du bien suprême et véritable dans le monde qui appartient au domaine des ténèbres, de la matière et du mal. Le mal n'est donc pas seulement une privation du bien, comme le pense W.A. Löhr ${ }^{40}$, mais il est le contraire du bien, dans un système à la fois métaphysique et physique fondé sur une séparation radicale entre le bien et le mal. La particularité de ce système consiste dans la superposition de plusieurs couples de contraires: l'intelligible et le sensible, l'âme et le corps, l'esprit et la matière, la lumière et les ténèbres.

La mention du bien "conçu au commencement», suivie de l'explication selon laquelle la lumière a permis aux créatures d'engendrer, fait penser au Prologue de l'évangile selon Jean. Dans ce texte, la lumière, qui est un autre nom du Logos de Dieu, est d'abord présentée comme celle qui «brille dans les ténèbres, et les ténèbres ne l'ont pas saisie» (Jn 1, 5); elle est "dans le monde» et «le monde fut par elle» (Jn 1, 3. 10). Cette "lumière véritable éclaire tout homme qui vient dans le monde» et n'est par reconnue par le monde, ni accueillie par les siens (Jn 1, 9-11). Sans que nous puissions signaler des points communs autres que ceux-ci, nous pensons plausible l'hypothèse selon laquelle le texte cité par Hégémonius serait un fragment de l'exégèse platonisante du

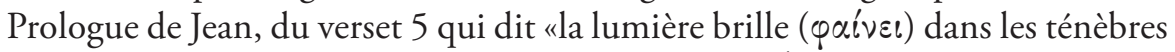

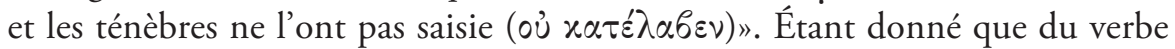

39. Acta Archelai 67, 10-12: Cum ergo nequiores de meliore sumpsissent non veram lucem, sed speciem quandam lucis atque enfasin, ... boni raptiva mutatione traxerunt. Unde nec perfectum bonum est in hoc mundo, et quod est valde est exiguum, quia parum fuit etiam illud quod initio conceptum est. Verum tamen per hoc ipsum exiguum lucis, immo potius per speciem quandam lucis, creaturae valuerunt generare similitudinem perferentem ad illam, quam de luce conceperant, permixtionem. Et haec est ista, quam cernimus, creatura. (éd. C.H. Beeson, pp. 96-97; trad. française S. Pétrement, Le dualisme, op. cit., pp. 191-192). L'édition et la traduction allemande de W.A. Löhr dans Basilides und seine Schule, op. cit., pp. 219-221.

40. W.A. Löhr en conclut que le mal et l'imperfection du monde n'est pas un principe au sens propre, mais l'absence du bien ou du meilleur (privatio boni); voir W.A. Löhr, Basilides und seine Schule, op. cit., p. 232. 
$\varphi \alpha i v \omega$ dérive $\varepsilon^{\prime} \mu \varphi \alpha \sigma \iota \varsigma$, tandis que $\chi \alpha \tau \alpha \lambda \alpha b^{\prime} \alpha \nu \omega$ veut dire saisir, retenir, s'emparer, la référence au Prologue pourrait justifier certains choix de vocabulaire qui nous ont surpris chez Basilide. Le dernier argument en faveur de notre hypothèse est l'identification entre les ténèbres et le monde, suggérée dans le Prologue de manière tout aussi rapide que dans le fragment basilidien. Le Logos étant identique à la lumière, nous obtenons les parallèles suivants: "lumière brille dans les ténèbres» $(1,5)$ et «il était dans le monde» $(1,10)$, puis «les ténèbres ne l'ont pas retenue» $(1,5)$ et «le monde ne l'a pas reconnu» $(1,10)$ suivi de «les siens ne l'ont pas accueilli» $(1,11)$. Dans l'hypothèse où Basilide commente le texte du Prologue, les termes "ténèbres», «monde» et "hommes» sont utilisés pour décrire la même réalité sensible, opposée à la lumière. C'est un argument supplémentaire pour dire que la matière dont parle Basilide est un autre nom donné aux ténèbres et non le produit de la rencontre de celles-ci avec le regard de la lumière.

Les échanges dramatiques entre la lumière et les ténèbres aboutissent à une conclusion particulièrement pessimiste: «la créature que nous voyons» possède la vie grâce à une apparence de l'apparence de la lumière, obtenue de force par les plus méchants. Une conclusion pareille est digne d'être posée à la base de l'anthropologie de Basilide, fondamentalement pessimiste, comme le montrent les fragments cités par Clément d'Alexandrie dans le sixième Stromate. Pour laver le Dieu créateur du moindre soupçon de responsabilité du mal dans le monde, Basilide attribue tous les maux aux péchés de ses créatures. Si les hommes souffrent, c'est parce qu'ils sont tous mauvais et «il ne faut pas [leur] imputer l'absence du péché»:

Comme celui qui désire l'adultère est adultère, même s'il n'arrive pas à le consommer, et que celui qui désire commettre un meurtre est déjà assassin, même s’il ne peut pas tuer, de même aussi quand je vois souffrir l'homme irréprochable dont je parle, même s'il n'a fait aucun mal, je le dirai mauvais par sa propension au péché. ${ }^{41}$

Cet autre passage basilidien témoigne de la même difficulté constatée au cours de notre lecture du passage cité par Hégémonius: Basilide sépare le bien et le mal, dans un effort de les opposer radicalement. Mais la plus grande difficulté qu'il rencontre vient du fait que dans ses doctrines de référence - le christianisme et le platonisme - le principe du bien est souverain et n'a pas de contraire. En ce qui concerne le christianisme, la réponse donnée par Clément d'Alexandrie a déjà été étudiée ${ }^{42}$, tandis que la référence platonicienne de

41. Strom IV, 82, 2; éd. A. Van Den Hoek, trad. C. Mondésert, SC 463, Paris, Cerf, 2001, pp. 190-193. Le passage cité fait partie du fragment plus vaste Strom IV, 81-83, classé par W.A. Löhr comme Fragment 7; voir Basilides und seine Schule, op. cit., pp. 122-138.

42. Voir Strom IV, 84-88; 95-96. L'objet de la critique de Clément est une thèse radicale de Basilide, à savoir la conception du martyre comme une punition méritée pour les maux commis dans la vie antérieure. Tant l'argument de Basilide que la réponse de Clément ont 
Basilide a été prise en considération dans le contexte cosmologique. Or, nous venons de constater que l'idée du Bien de la République pourrait servir de modèle à Basilide dans sa manière de présenter la lumière, en tant que principe du bien. Toutes les distorsions par rapport à la métaphysique platonicienne viennent du fait que Basilide cherche à trouver le contraire du Bien. Mais le prix à payer est très élevé, parce que la lumière basilidienne n'est jamais vraiment séparée des ténèbres et, à cause de leurs affections mutuelles, n’obtiendra jamais le statut «au-delà de l'essence», qui est la marque de transcendance de l'Un-Bien néoplatonicien. Est-il possible de trouver une solution à cette difficulté?

\section{Une lecture cosmogonique alternative}

La lecture du passage basilidien cité par Hégémonius, en tant qu'inspiré de la République, n'est pas la seule interprétation possible. En fait, W.A. Löhr en propose une autre lecture, inspirée davantage du Timée (48e, 50c-53c), selon laquelle l'histoire des rapports entre la lumière et les ténèbres possède une visée cosmogonique. Pour plusieurs raisons, cette proposition mérite l'intérêt, même si - à notre avis - la principale préoccupation de Basilide est de donner une assise métaphysique à sa doctrine morale. Les éléments naturels y sont cependant présents, pas seulement à titre d'analogie, mais aussi à travers certains détails concernant les qualités physiques de la lumière et des ténèbres. Nous tenons donc l'interprétation cosmogonique pour importante, mais sans lui accorder une importance décisive.

L'interprétation cosmogonique du fragment des Actes renoue avec une certaine tradition dans les études basilidiennes qui date des temps de W. Bousset et E. de Faye et qui s'inspire de la présentation que Basilide lui-même fait de sa doctrine comme tirée des "questions que les barbares eux-mêmes se sont posées, concernant les biens et les maux et les opinions auxquelles ils sont parvenus sur tout cela.» ${ }^{43}$ La mention des "barbares» a orienté les recherches vers les doctrines dualistes orientales, telles le zoroastrisme, le manichéisme et même le bouddhisme ${ }^{44}$. G. Quispel oppose à cette tendance sa propre interprétation

été analysés et ont été objets de débats, voir P. Nautin, «Les fragments de Basilides sur la souffrance et leur interprétation par Clément d'Alexandrie et Origène», dans Mélanges d'histoire des religions offerts à H.-Ch. Puech, Paris, 1974, pp. 393-403; W.A. Löhr, Basilides und seine Schule, op. cit., pp. 145-152.

43. Acta Achelai 67, 7: Desinamus ab inani et curiosa varietate; requiramus autem magis quae de bonis et malis etiam barbari inquisierunt et in quas opiniones de his omnibus pervenerunt. (éd. C.H. Beeson, p. 96: trad. S. Pétrement dans, Le dualisme, op. cit., pp. 191-192). Voir le commentaire dans W.A. Löhr, Basilides und seine Schule, op. cit., pp. 233-249

44. W. Bousset (et avant lui A. Hilgenfeld) a attribué l'influence «barbare» au dualisme iranien (voir Hauptprobleme der Gnosis, Göttingen, 1907, pp. 93-96), tandis que J. Kennedy au bouddhisme (voir "Buddhist Gnosticism, the System of Basilides», The Journal of the Royal Asiatic Society [1902], pp. 377-415). Pour la polémique avec ces positions et avec 
de la doctrine basilidienne comme une forme de "gnose savante», issue du mélange du platonisme hellénistique et du christianisme alexandrin. Malheureusement, son interprétation du passage des Actes d'Achélaüs repose sur un malentendu concernant la nature des rapports entre la lumière et les ténèbres: il voit dans la lumière un principe féminin désireux de voir le visage des ténèbres, un principe masculin; cela a pour résultat de transformer leurs relations en une cosmogonie sur fond d'amour tragique ${ }^{45}$. Cette lecture a déjà fait l'objet de l'analyse critique de W.A. Löhr, qui toutefois en retient certains éléments, ceux qui lui permettent de mettre en relief le côté cosmogonique du texte.

Dans cette perspective, la lumière - principe du Bien - est inspirée de la figure du Démiurge du Timée, telle qu'elle résulte de la longue section consacrée à ses rapports avec la $\chi \tilde{\omega} \rho \alpha$, le réceptacle des Idées. Une autre source d'inspiration serait le Banquet, le mythe de la naissance d'Éros. Or, l'exégèse de ce mythe faite par Plutarque dans De Isis et Osiris, s'avère particulièrement éclairante et permet de réunir les thèmes de ces deux grands textes de Platon, susceptibles de façonner la cosmogonie de Basilide. Grâce à la comparaison entre le traité de Plutarque et le fragment basilidien, il devient possible d'établir une série d'analogies entre les couples: la lumière et les ténèbres chez Basilide, Isis et Osiris chez Plutarque, Poros et Pénia chez Platon. Placés dans une perspective cosmogonique, les personnages mythologiques, masculin et féminin, se transforment en principes contraires. Vu ainsi, le fragment de Basilide répond aux critères d'un mythe cosmogonique.

Cependant, la lecture attentive du traité de Plutarque dévoile quelques rapprochements mal ajustés. Selon Plutarque, Isis et Osiris sont des bons démons qui, à cause de leurs vertus, passent au rang des dieux. Sur le plan cosmique, Osiris correspond à l'élément humide et Isis représente la matière. Or celle-ci n'est pas le principe du mal, parce que - toujours selon la réinterprétation plutarquienne du mythe - le mal est représenté par Typhon, le démon malfaisant qui symbolise tout ce qui est aride, igné et desséché. On constate déjà que la comparaison avec le texte de Basilide commence à se fissurer.

Mais les différences ne s'arrêtent pas là, parce que Plutarque est également confronté à la question de l'origine du mal. À la différence de Basilide, il donne au mal d'abord un sens moral et refuse d'attribuer ses origines à un principe

une dépréciation de la pensée de Basilide, voir G. Quispel, «L'homme gnostique», art. cit., pp. 89-96. Sur les interprétations "orientalistes» de Basilide postérieures à l'article de Quispel, voir W.A. Löhr, Basilides und seine Schule, op. cit., pp. 233-234.

45. "Voici les motifs de ce texte: la lumière est désirée, elle est curieuse: ça doit être une femme, la Vierge de la Lumière ou une personnification analogue; cette femme devient victime de sa curiosité, comme la Psyché, qui dans l'allégorie savante d'Apulée, voulait voir la face d'Amor. [...] cette femme, qui par suite de sa curiosité, tombe dans les ténèbres de la matière [...] c'est un mythe platonicien sur la chute de l'âme humaine.» G. Quispel, «L'homme gnostique», art. cit., p. 95. 
physique $^{46}$. Néanmoins, il aborde dans une longue digression l'existence de principes contraires qui sont des dieux rivaux, causes respectives du bien et du mal. Plutarque passe ainsi en revue plusieurs opinions et cite, entre autres, Zoroastre qui associait la lumière au bien et les ténèbres au mal ${ }^{47}$. Mais ce sont les opinions des philosophes de la Grèce auxquelles il accorde la plus grande importance:

Les pythagoriciens usent d'une grande variété des termes: ils disent pour désigner le principe bienfaisant: l'Un, la Limite, l'Immobile, le Droit, l'Impair, le Carré, l'Egal, le Dextre, le Brillant, et pour désigner le principe malfaisant: la Dyade, l'Illimité, le Mobile, le Courbe, le Pair, l'Oblong, l'Inégal, le Sénestre, le Sombre, et ils y voient les principes de la génération. Anaxagore oppose l'Esprit et l'Illimité, Aristote la Forme et la Privation. Quant à Platon, il emploie souvent des termes obscurs et enveloppés et appelle les deux principes opposés respectivement le «Même» et l'«Autre». 48

Eu égard à cette doxographie, le dualisme de Basilide perd beaucoup de son allure barbare. Plutarque applique alors les positions dualistes au mythe qu'il interprète et conclut qu'Osiris représente le principe du Bien, parce qu'il est toute forme d'excellence qui se présente sur la terre: l'ordre, la stabilité, la santé, etc., tandis que Typhon est le principe malfaisant qui se manifeste dans tout ce qui est déréglé, instable et malsain (371b-c). Si le rapport entre Osiris et Typhon est assez évident, la place d'Isis ne l'est pas autant. Plutarque présente la déesse comme «nature» et plus loin comme «matière»:

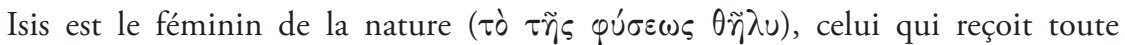
génération, d'où les noms de "nourrice», d'«universel réceptacle» que lui donne Platon (cf. Timée 49a-51a). Elle aime d'un amour inné l'Être premier, le souverain de l'Univers, qui n'est autre que le Bien, elle le désire et le recherche, alors qu'elle fuit et repousse ce qui est du domaine du Mal. Si elle est le lieu et la matière ( $\chi \omega \dot{\omega} \rho \alpha$

46. «Car il ne faut pas placer dans les corps inanimés les principes de l'univers, comme Démocrite et Epicure, il ne faut pas non plus postuler, avec les Stoïciens, une Raison ou Providence unique, souveraine maîtresse de toutes choses, qui serait seule à agir sur une matière dépourvue de qualités. En effet, aucune forme de mal ne saurait apparaître là où Dieu est responsable de tout, aucune forme de bien là où il n'est responsable de rien.» $D e$ Isis et Osiris, 45 (369a), trad. Ch. Froidefond, p. 217.

47. Voir Isis et Osiris 46-47 (369d-370c).

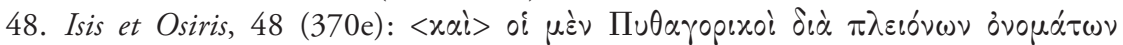

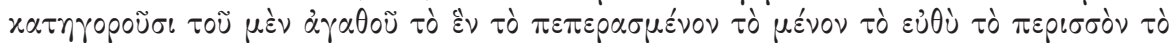

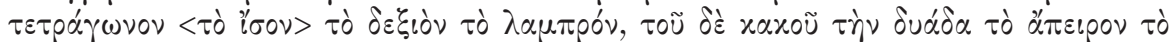

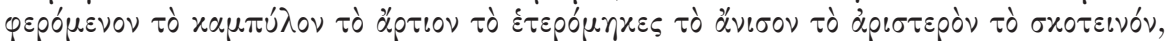

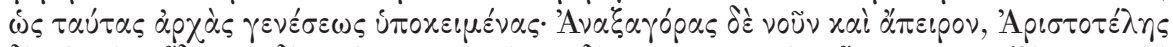

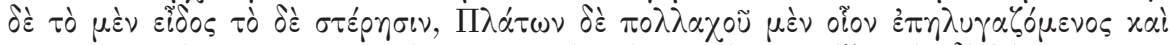

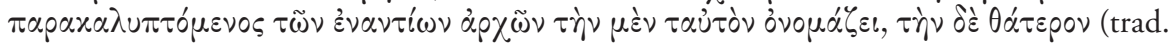
Ch. Froidefond, p. 221). Le texte fait allusion à Timée 35a. 


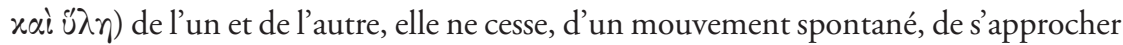
du principe du Bien; c'est à lui qu'elle s'offre pour qu'il procrée en elle et sème en elle les émanations qui portent sa ressemblance. ${ }^{49}$

La description que Plutarque fait d'Isis-nature-matière est très éloquente et, par de nombreux traits, elle ressemble aux ténèbres-matière de Basilide. Mais il ne faut pas oublier qu'Isis n'est pas le principe du mal et, par conséquent, son personnage ne répond pas à la préoccupation fondamentale du dualisme basilidien. En outre, Isis n'est pas matière au sens propre du terme; et tant qu'elle représente toutes les ambiguïtés de la $\chi \omega ́ p \alpha$ platonicienne, Plutarque l'appelle à la fois nature, lieu et matière. Comme beaucoup de ses condisciples, Plutarque se sert d'un terme aristotélicien, «matière» ( $\left.\mho^{\prime} \lambda \eta\right)$, pour expliquer les concepts platoniciens, même s'il sait, par ailleurs, que Platon ne l'a pas utilisé dans ce sens ${ }^{50}$. Grâce à l'allusion à la longue section du Timée 49a-51a, il est plus facile de repérer les éléments supposés faire le lien entre l'interprétation cosmologique du mythe d'Isis et d'Osiris et le texte de Basilide. En fait, après avoir présenté Isis comme une sorte de principe féminin, Plutarque se réfère au Timée 50b-e, où Platon explique ce qu'est la $\chi \omega ́ p \alpha$. L'essentiel de son explication est donné en 50c7-e4 où il appelle le modèle "père», le réceptacle «mère» et «enfant» ce qui procède d'eux ${ }^{51}$. Sous la plume de Plutarque, ces trois termes vont se transformer en Osiris, Isis et Horus, où Osiris est le principe du bien, Isis la nature et Horus le monde sensible. La Nature suit le Bien d'un mouvement spontané et ainsi elle fuit le mal. Horus représente le monde sensible, parce qu'il est enfanté par Isis, à la fois nature et matière première, à l'image d'Osiris, principe suprême du bien. Isis ne représente pas le mal, mais elle est, pour ainsi dire, suspendue entre le bien et le mal, entre l'intelligible et le sensible; elle produit selon ce qu'elle reçoit et ses «enfants» peuvent «tomber

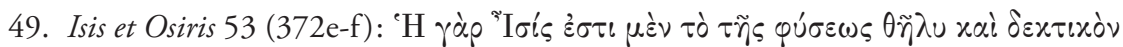

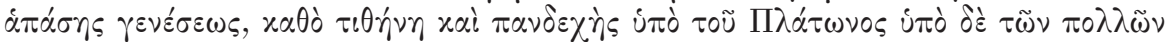

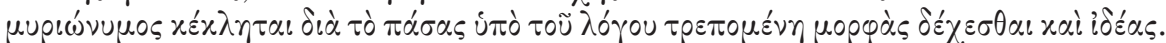

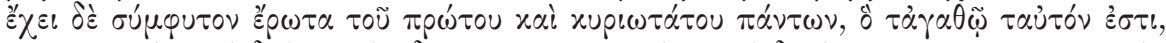

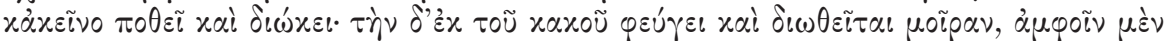

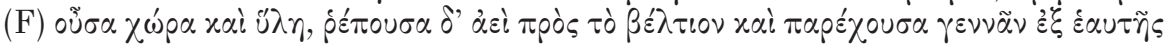

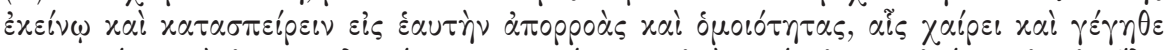

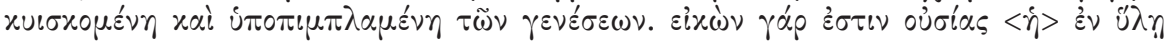

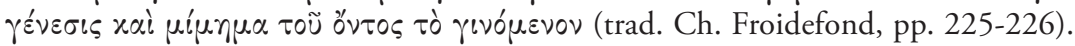

50. Sur les usages du terme ú' $\lambda \eta$ à côté de $\chi(\omega) \rho \alpha$, voir Alcinoos, Didascalicos VIII; Apulée, De Plat. 191-192; Plutarque, De an. procr. in Tim 1013c; De deforac. 414f; Calcidius, Comm in Tim, p. 273; 309. Sur la conception de la matière chez les médio-platoniciens, voir J.C.M. van Winden, Calcidius on Matter: His Doctrine and Sources, Leyde, 1965.

51. Voir Timée 50c7-e4: «Et tout naturellement il convient de comparer le réceptacle à une mère, le modèle à un père, et la nature qui tient le milieu entre les deux à un enfant", trad. L. Brisson, pp. 149-150. Plutarque se réfère explicitement à ce texte dans Isis et Osiris, $56(373 \mathrm{e})$. 
au pouvoir du principe de désordre et de perturbation» (54, 373a). Ainsi

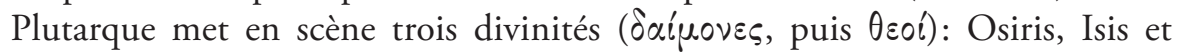
Horus - qu'il compare avec les personnages de la mythologie grecque et les lectures qu'en ont faites les philosophes. Il construit ainsi une figure triangulaire formée du bien-intelligible, de la nature-matière et du monde sensible. Aucun des trois ne représente le mal qui, redisons-le, est relégué au domaine éthique, pas à ceux de la métaphysique ou de la physique.

La comparaison avec le texte de Basilide montre plutôt que sa conception des ténèbres-matière ne peut être influencée que très modestement par la notion de $\chi \omega \dot{\omega} \rho \alpha$, sinon au prix d'une très importante déformation de ce concept platonicien qui alors devient non seulement synonyme du sensible, mais encore du mal. Les rapports entres Osiris, Isis et Horus permettent également de prendre mieux conscience du fait que la question de l'origine du bien ou du mal dans le monde n'est pas identique à la question de l'origine du monde.

Pour terminer l'examen de l'interprétation cosmogonique du passage des Actes, revenons sur la référence au mythe de la naissance d'Éros évoqué par Plutarque comme un exemple de couple formé par le principe du Bien et la matière préexistante ${ }^{52}$ :

Poros n'est ici autre que l'objet suprême de l'amour et du désir, l'être parfait qui se suffit à lui-même. Pénia est le nom que Platon a donné à la matière, en elle-même dépourvue du Bien, mais qui s'ouvre à lui et sans cesse le désire et en prend sa part. Et le fruit de leur union, le monde, autrement dit Horus, n'est ni éternel, ni inaffectible, ni indestructible, mais toujours renaissant... ${ }^{53}$

Cette exégèse du Banquet ne correspond que partiellement à la dynamique des rapports entre la lumière et les ténèbres de Basilide, parce qu'ici encore la matière ne représente pas le mal, mais le manque de Bien. Le passage que nous venons de citer montre, tout comme le mythe précédent, que dans les deux cas Plutarque met en place les structures ternaires: Osiris-Isis-Horus et Poros-Pénia-Éros. En faisant ainsi, il suit de près le passage du Timée 50b-e,

52. Pour étayer son argumentation Plutarque utilise plusieurs dialogues de Platon; outre le Timée, déjà mentionné, on trouve les allusions aux Lois (896e) et à la République (546b), mais le point culminant est la comparaison avec le mythe de la naissance d'Éros raconté dans le Banquet (203b-c). Le résumé du passage des Lois (896e) se trouve en Isis et Osiris 48 (371f); voir Notes complémentaires dans Plutarque, Euvres morales V, 2 - Isis et Osiris, trad. Ch. Froidefond, Paris, Les Belles Lettres, 1988, p. 298. Le passage de la République (546b) est commenté en Isis et Osiris 56 (373f-374a); voir Notes complémentaires, dans Plutarque, Euvres morales V, 2, op.cit., pp. 304-305.

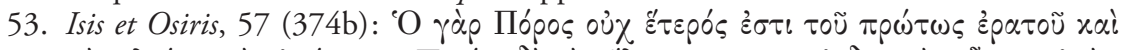

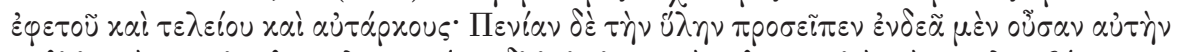

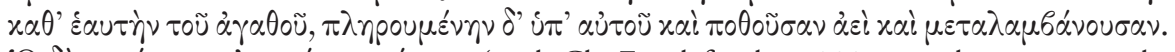

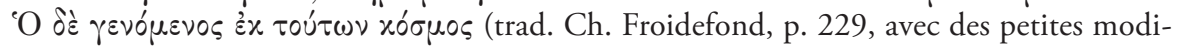
fications). Voir aussi Notes complémentaires, pp. 305-306. 
essentiel pour la cosmogonie platonicienne. Or Basilide raisonne autrement, probablement parce qu'il ne cherche pas à expliquer comment le monde sensible est venu à l'existence, mais pourquoi ce monde est séparé du bien. Dans sa perspective, l'opposition entre le masculin et le féminin est juste une opposition de plus; elle n'a pas vocation à être déterminante pour les rapports entre les principes. Cela mène à la conclusion que la doctrine exposée dans les Actes d'Archélaüs non seulement n'est pas une cosmogonie, mais qu' elle ne peut pas en être une, parce que la rencontre entre la lumière et les ténèbres ne produit rien d'autre que la «couleur de lumière» sur la matière, soit une illusion d'optique. Basilide insiste trop lourdement sur le fait qu'il n'y a aucun mélange entre la lumière et les ténèbres pour qu'on puisse supposer que leur rencontre ait produit une troisième réalité.

La lecture des textes de Plutarque et du Timée permet de faire l'état des écarts qui séparent Basilide de la tradition platonicienne, et qui, en même temps, témoignent de la grande originalité de sa pensée. On constate d'abord que la visée de Basilide est plus vaste que la cosmogonie. Il ne s'intéresse pas à la genèse du monde sensible ou intelligible, mais aux raisons de la présence du mal et de l'absence du bien dans la vie des hommes. Cette perspective explique, à notre sens, pourquoi il est si difficile de trouver des modèles platoniciens adéquats, et plus encore lorsque l'on cherche du côté des modèles cosmogoniques. Même en adoptant une perspective métaphysique, la principale difficulté à la laquelle se heurte Basilide vient du soi disant dualisme platonicien, ainsi que $\mathrm{du}$ fonctionnement des notions du bien et du mal chez Platon. Indéniablement, Platon utilise les structures dualistes, parmi lesquelles la plus importante est l'opposition entre l'intelligible et le sensible, et Basilide exploite avant tout cette opposition-là. Mais en même temps, l'idée platonicienne du Bien est élevée au rang de première parmi les idées, sans qu'une quelconque «idée du mal» lui soit opposée. C'est là que Basilide déploie son ingéniosité pour fabriquer un principe du mal capable de faire face à son principe du bien - la lumière - inspiré du chapitre VI de la République. Mais, comme nous l'avons déjà remarqué, il ne peut pas maintenir la souveraineté du Bien platonicien dans un univers gouverné par deux principes contraires.

\section{Et après? - une solution radicale}

Plus haut nous avons posé la question - est-il possible de trouver une solution à la difficulté rencontrée par Basilide? Il semble que oui, mais celle-ci ne viendra qu'un siècle après. Il est possible que la notice d'Hippolyte' de Rome raconte une tentative de remédier aux contradictions engendrées par une interprétation dualiste de la métaphysique platonicienne. 
De prime abord, la doctrine de Basilide, présentée longuement dans la Réfutation, ne ressemble en rien à celle du fragment des Actes d'Achélaüs ${ }^{54}$. 'Hippolyte' donne toutefois l'impression d'être plutôt bien renseigné, parce qu'il mentionne Basilide parmi les "hérétiques» les plus anciens (Cérinthe, Cerdon, Marcion, les ébionites, Carpocrate, Satornil, Ménandre), proches des temps apostoliques. Comme il parle aussi du fils de Basilide, Isidore, et des basilidiens, on suppose qu'il connaît plutôt une forme tardive de cette doctrine, probablement influencée par la rencontre avec le valentinisme ${ }^{55}$. Néanmoins, on a du mal à le croire lorsqu'il tente un rapprochement entre la théologie de Basilide et les catégories d'Aristote. En effet, 'Hippolyte' consacre de longues pages à exposer de manière succincte - et donc très superficielle - ce qu'est le genre, l'espèce, l'individu et la substance ${ }^{56}$. L'erreur ou la mauvaise foi du polémiste chrétien se manifeste dans sa manière systématique de confondre la chose et le nom de la chose, la substance et les catégories permettant de la saisir conceptuellement.

Sans entrer dans l'analyse de la présentation caricaturale qu'Hippolyte' fait de la doctrine basilidienne, limitons-nous à quelques-uns de ses traits essentiels, facilement repérables. Il s'agit d'une courte section (Refut. VII, 20-21) qui a déjà attiré l'attention des chercheurs en tant qu'expression de la théologie négative, susceptible à ce titre d'influencer la conception de l'Un de Plotin ${ }^{57}$. Ce qu'on remarque en premier dans les citations de Basilide rapportées dans

54. Hippolyte, Refut VII, 20-27; X, 14; voir W.A. Löhr, «Basilides», dans DGWE, pp. 167-168; W.A. Löhr, Basilides und seine Schule, op. cit., pp. 284-323.

55. Nous laissons de côté l'étrange doctrine de trois Filialités qui semble être assez proche du valentinisme; voir Refut VII, 22-27. Déjà dans la notice d'Irénée de Lyon, la doctrine de Basilide prend quelques traits valentiniens; Irénée expose sa conception de successio haereticorum $\left(\delta\left\llcorner\alpha \delta \circ \chi \eta^{\prime}\right)\right.$, dans laquelle Basilide figure comme l'un des ancêtres des valentiniens; voir Irénée de Lyon, AdvHaer I, 23-27; A. Le Boulluec, op. cit., pp. 85-86; 163-166; W.A. Löhr, Basilides und seine Schule, op. cit., pp. 257-262. En conséquence, la doctrine qu'Irénée attribue à Basilide ressemble à celle de Valentin. Selon cette notice, du Père inconnu provient Nous, après lequel viennent Logos, Phronesis, Sophia et Dynamis. De ces dernières viennent les Vertus, les Archontes et les Anges. Les émanations des Anges et des Archontes continuent, jusqu'à la formation des 365 cieux. Irénée mentionne toutefois certains points de la doctrine basilidienne, notamment le refus du martyre - parce que sur la croix est mort Simon de Cyrène et Jésus a trompé ainsi les Archontes; voir Irénée, AdvHaer I, 24, 3-4. Pour le commentaire, voir W.A. Löhr, Basilides und seine Schule, op. cit., pp. 255-273.

56. Voir Refut VII, 14-19. Dans cette section 'Hippolyte' résume les passages suivants: Cat 2, 1a20. 1b6; Cat 5, 2a11.14-27.3a35. On trouve aussi des expressions ou des phrases provenant des traités De anima, Métaphysique $\Lambda$ et du traité pseudo-aristotélicien De mundo. Voir l'édition de P. Wendland, pp. 191-195; celle de M. Marcovich, pp. 280-286.

57. Le premier qui a signalé l'importance de ce passage et de son interprétation dans le sens de la théologie négative est J. Whittaker, «Basilides on the Ineffability of God», HThR 62 (1969), pp. 367-371. Ensuite, le rapprochement avec l'Un de Plotin est proposé par M. Jufresa Muños, «Basilides, a path to Plotinus», Vigiliae Christianae 35 (1981), pp. 5-15. 
la Réfutation, c'est un effort d'exprimer le «rien» de manière absolue. Il commence par dire que Dieu est inexprimable, mais au point de ne même pas pouvoir être nommé ainsi: "ce qui est inexprimable ne peut même pas être

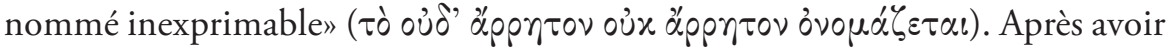
nié la possibilité de lui attribuer un nom, l'auteur basilidien a probablement poursuivi sa réflexion théologique en rejetant toutes les catégories permettant de saisir la substance de Dieu, jusqu'à la négation de cette substance elle-même

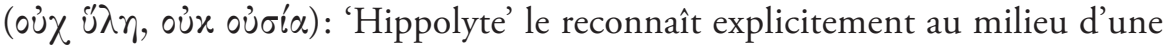
longue série de moqueries terminée par l'aveu que Dieu n'est absolument aucun être qu'on puisse nommer ou percevoir par les sens ou par l'intelligence. Évidemment, le basilidien lui refuse également toute forme de volonté, de pensée et de sensation. Au terme de cette analyse, le Dieu des basilidiens est «le Dieu non existant» ( $\langle\dot{\delta}\rangle$ oủx ¿̀े $\theta \varepsilon o ́ \varsigma)$, ce qui - selon 'Hippolyte' serait un

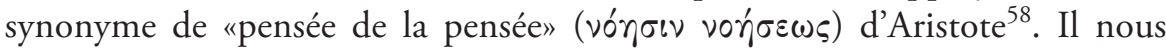
semble cependant que l'inspiration basilidienne vienne plutôt de la célèbre formule d'Ex. 3, 14 où Dieu se présente comme ó $\omega^{\prime} \nu$. Ainsi, les négations basilidiennes visent l'être de Dieu.

Inutile de s'acharner sur le traitement infligé par 'Hippolyte' à la doctrine aristotélicienne, notre objectif étant la transformation du principe du Bien chez Basilide. Or, il procède par des négations de tout ce qui est relatif au sensible, à la matière, à la substance. La logique qui dirige cette théologie est toujours tributaire du dualisme primitif qui a caractérisé la doctrine exprimée dans les Actes d'Archélaüs. Nous avons constaté que la plus grande difficulté rencontrée par Basilide dans son effort de prendre le Bien platonicien pour modèle de son principe du Bien, consistait dans une quasi-impossibilité d'affranchir ce Bien de toute relation avec le sensible, à savoir avec le Mal. Même après avoir établi une chaîne d'intermédiaires (miroir, reflet, couleur, rapt), il n'en reste pas moins qu'un souvenir lointain du Bien demeure toujours dans le monde censé être fondamentalement mauvais. La solution trouvée surprend par son radicalisme: afin d'assurer sa transcendance, les basilidiens suppriment le nom et l'être même du Bien. Il n'est plus «Bien», parce qu'il n'a aucun nom capable de le nommer. Il n'est plus "au-delà de l'essence»: il est sans essence, voire sans être. La seule chose qui reste, c'est une expression de transcendance divine, la plus radicale

Son étude a le mérite d'offrir une analyse très fouillée des termes utilisés par Basilide, comparés aux doctrines philosophiques de son époque.

58. Voir Refutatio VII, 20-21. Signalons que dans le Traité 33 Plotin accuse certains gnostiques de professer une théorie de deux intellects, dont «l'un pensée et l'autre pense

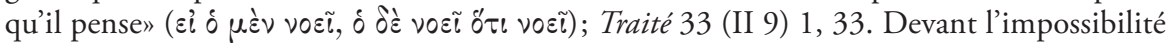
de trouver une doctrine correspondante à cette description, notre seule piste est l'accusation formulée par 'Hippolyte' à l'encontre des basilidiens, mais à condition de prouver que ceux-ci ont réellement fait usage de l'expression aristotélicienne "pensée de la pensée»

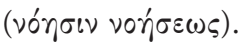


dans les doctrines chrétiennes de l'Antiquité. Cependant, Basilide se sert de son concept de théologie de Dieu sans être pour expliquer que la création vient du néant absolu, d'un nibil sans être. Maintenant, il n'y a aucune possibilité de lien entre ce Dieu et le sensible, le monde et, enfin, avec le mal. C'est ainsi que les exigences d'une métaphysique dualiste reçoivent enfin une solution satisfaisante - si on peut parler d'une solution, étant donné que le principe du Bien a perdu l'existence.

Avons-nous donc obtenu la réponse à la question initiale: qu'est devenu le principe du Bien, au terme de ces longs efforts pour lui trouver un contraire? Les efforts de Basilide des Actes d'Archélaüs ont montré à quel point la métaphysique platonicienne - pourtant taxée de dualiste! - résiste à une lecture dualiste radicale. L'impossibilité de donner un contraire à l'idée du Bien avait pour première conséquence une élaboration de la chaîne des intermédiaires, ce qui coïncide peut-être avec un rapprochement avec le valentinisme, dont témoignent Irénée de Lyon et la curieuse doctrine de trois Filialités relatée par 'Hippolyte' dans la suite de la notice. Mais la solution ultime et radicale vient d'une proposition théologique d'une extrême audace, à savoir d'un principe sans existence, ce qui, en fin du compte, constitue la seule solution crédible pour une métaphysique dualiste. 